\title{
ON THE AVERAGE ORDER OF IDEAL FUNCTIONS AND OTHER ARITHMETICAL FUNCTIONS
}

\author{
BY BRUCE C. BERNDT
}

Communicated by Paul T. Bateman, July 15, 1970

\begin{abstract}
We consider a large class of arithmetical functions generated by Dirichlet series satisfying a functional equation with gamma factors. We state a general O-theorem for the average order of these arithmetical functions and apply the result to ideal functions of algebraic number fields.
\end{abstract}

Landau [4] and Chandrasekharan and Narasimhan [3] have proved O-theorems for the average order of a large class of arithmetical functions. The method of proof uses finite differences and is due to Landau. Often, it is desired to have an O-theorem where the error term is a function of a certain parameter, which is the discriminant, for example, in the case of an algebraic number field. We state here a general O-theorem of this type. The method of proof is a slight modification of Landau's mentioned above.

We briefly indicate the arithmetical functions under consideration. For a more complete description see [3].

Let $\{a(n)\}$ and $\{b(n)\}$ be two sequences of complex numbers not identically zero. Let $\left\{\lambda_{n}\right\}$ and $\left\{\mu_{n}\right\}$ be two strictly increasing sequences of positive numbers tending to $\infty$. Put $s=\sigma+i t$ with $\sigma$ and $t$ both real. We assume that

$$
\varphi(s)=\sum_{n=1}^{\infty} a(n) \lambda_{n}^{-s} \text { and } \psi(s)=\sum_{n=1}^{\infty} b(n) \mu_{n}^{-s} ;
$$

each converge in some half-plane and satisfy the functional equation

$$
\Delta(s) \varphi(s)=\Delta(r-s) \psi(r-s),
$$

where $r$ is real and

$$
\Delta(s)=\prod_{\nu=1}^{N} \Gamma\left(\alpha_{\nu} s+\beta_{\nu}\right)
$$

where $\alpha_{\nu}>0$ and $\beta_{\nu}$ is complex, $\nu=1, \cdots, N$.

In the sequel $A$ always denotes a positive number not necessarily the same with each occurrence.

AMS 1969 subject classifications. Primary 1043, 1065; Secondary 1041.

Key words and phrases. Functional equation with gamma factors, arithmetical function, ideal function, average order, error term. 
For $q \geqq 0$, let

$$
A_{q}(x)=\frac{1}{\Gamma(q+1)} \sum_{\lambda_{n} \leq x} a(n)\left(x-\lambda_{n}\right)^{q} .
$$

Let

$$
Q(x)=\frac{1}{2 \pi i} \int_{c} \frac{\phi(s) x^{s}}{s} d s,
$$

where $C$ is a cycle encircling all of the integrand's singularities. We shall assume that $\lambda_{n}=\lambda \lambda_{n}^{*}$ and $\mu_{n}=\lambda \mu_{n}^{*}$ where $\lambda>0$ is a constant for the particular pair of Dirichlet series $\varphi$ and $\psi$, and where $\lambda_{n}^{*}$ and $\mu_{n}^{*}$ are not functions of $\lambda$. E.g., if we consider the zeta-function of an algebraic number field $K$, then $\lambda=d^{-1 / 2}$, where $d$ is the modulus of the discriminant of $K$. Define

$$
\begin{gathered}
A_{q}^{*}(x, \lambda)=A_{q}(\lambda x)=\frac{\lambda^{q}}{\Gamma(q+1)} \sum_{\lambda_{n}{ }^{*} \leq x} a(n)\left(x-\lambda_{n}^{*}\right)^{q}, \\
Q^{*}(x, \lambda)=Q(\lambda x),
\end{gathered}
$$

and the error term

$$
P^{*}(x, \lambda)=A_{0}^{*}(x, \lambda)-Q^{*}(x, \lambda) .
$$

Let $\sigma_{a}^{*}$ denote the abscissa of absolute convergence of $\psi$. From [3, p. 111$], \sigma_{a}^{*} \geqq \frac{1}{2} r+1 /(4 \alpha)$, where

$$
\alpha=\sum_{\nu=1}^{N} \alpha_{p}
$$

The starting point for our investigation is an identity of Chandrasekharan and Narasimhan [3, p. 99]. If $m$ is a sufficiently large positive integer and $x>0$,

$$
A_{m}(x)-S_{m}(x)=\sum_{n=1}^{\infty} \frac{b(n)}{\mu_{n}^{r+m}} I_{m}\left(\mu_{n} x\right),
$$

where $S_{m}(x)$ arises from the singularities of $\Gamma(s) \varphi(s)$ and $d^{m} S_{m}(x) / d x^{m}$ $=Q(x)$, and where

$$
I_{m}(x)=\frac{1}{2 \pi i} \int_{c_{m-i \infty}}^{c_{m}+i \infty} \frac{\Gamma(r-s) \Delta(s)}{\Gamma(m+1+r-s) \Delta(r-s)} x^{r+m-s} d s,
$$

where $c_{m}=(\alpha r+m) / 2 \alpha-\epsilon, 0<\epsilon<1 / 4 \alpha$. We choose $\epsilon$ so that the path of integration contains no poles of the integrand.

We now state the 
THEOREM. Suppose that there is a positive integer $m$ such that (2) holds, $r / 2+1 / 4 \alpha+m / 2 \alpha>\sigma_{a}^{*}$, and the integrand of $I_{m}(x)$ has no poles for $c_{0} \leqq \sigma \leqq c_{m}$. Suppose that the singularities of $\varphi$ (if any) are at most poles (finite in number). Assume that there exist real constants $a, b, c, d$, $a^{\prime}, b^{\prime}, c^{\prime}$ and $d^{\prime}$ and $a$ function $f(\lambda)$ such that

and

$$
A_{0}^{*}(x, \lambda), \sum_{\mu_{n}^{*} \leq x}|b(n)|=O\left(x^{a} \lambda^{b} \log ^{c} x|\log \lambda|^{d}\right)
$$

$$
Q^{*}(x, \lambda)=O\left(x^{a^{\prime}} \lambda^{b^{\prime}} \log ^{c^{\prime}} x|\log \lambda|^{d^{\prime}}\right)+f(\lambda),
$$

uniformly as $x$ tends to $\infty$ and $\lambda$ tends to 0 . Let

$$
\rho=\frac{b-b^{\prime}+r-2 a}{2 \alpha a-r \alpha+1 / 2} \quad\left(2 \alpha a-r \alpha+\frac{1}{2} \neq 0\right),
$$

and $z=\left(x^{\eta} \lambda^{1 / \alpha-\rho}\right)^{2 \alpha}$, where $\eta \geqq 0$ and $\alpha$ is given by (1). Define

$$
\begin{aligned}
E(x, \lambda)= & x^{a^{\prime}-1 / 2 \alpha-\eta \lambda^{b^{\prime}+\rho}} \log \mathrm{c}^{c^{\prime}} x|\log \lambda|^{d^{\prime}} \\
& +x^{r / 2-1 / 4 \alpha+\eta\left(2 \alpha a-r \alpha-\frac{1}{2}\right)} \lambda^{b^{\prime}+\rho} \log ^{c} z|\log \lambda|^{d} .
\end{aligned}
$$

Assume that, for $x^{1 / 2 \alpha+\eta} \leqq A \lambda^{\rho}$,

$$
x^{a} \lambda^{b} \log ^{c} x|\log \lambda|^{d}=O\{E(x, \lambda)\},
$$

and that, for $x^{1 / 2 \alpha+w} \geqq A \lambda^{p}$,

$$
f(\lambda)=O\{E(x, \lambda)\}
$$

uniformly as $x$ tends to $\infty$ and $\lambda$ tends to 0 . Then, if $\sigma_{a}^{*}>r / 2+1 / 4 \alpha$,

$$
P^{*}(x, \lambda)+f(\lambda)=O\left(\sum_{x<\lambda_{n}^{*} \leq x+O\left(x^{1-1 / 2 \alpha-\eta \lambda \rho)}\right.}|a(n)|\right)+O\{E(x, \lambda)\},
$$

uniformly as $x$ tends to $\infty$ and $\lambda$ tends to 0 . Furthermore, if $a(n) \geqq 0$,

$$
P^{*}(x, \lambda)+f(\lambda)=O\{E(x, \lambda)\} \text {. }
$$

If $\sigma_{a}^{*}=r / 2+1 / 4 \alpha$, we have the same results as above, except that an additional factor of $\log z$ must be placed in the second term defining $E(x, \lambda)$.

EXAMPLE. Let $K$ be an algebraic number field of degree $n=r_{1}+2 r_{2}$, where $r_{1}$ is the number of real conjugates and $2 r_{2}$ the number of imaginary conjugates in $K$. The Dedekind zeta-function for $K$ is defined by

$$
\zeta_{K}(s)=\sum_{\nu=1}^{\infty} F(\nu) \nu^{-*}, \quad \sigma>1
$$


where $F(\nu)$ is the number of nonzero, integral ideals of norm $\nu$ in $K$. Furthermore, $\zeta_{K}(s)$ satisfies the functional equation

$$
\xi(s)=\xi(1-s),
$$

where $\xi(s)=B^{\varepsilon} d^{\delta / 2 s} \Gamma^{r_{1}}\left(\frac{1}{2} s\right) \Gamma^{r_{2}}(s) \zeta_{K}(s)$, where $B$ is a constant depending only on $n$, and $d$ is the modulus of the discriminant of $K$. In the notation of our theorem we have $\alpha=\frac{1}{2} n$ and $\lambda=d^{-1 / 2}$. Also, it is well known that

$$
Q^{*}\left(x, d^{-1 / 2}\right)=Q^{*}\left(x, d^{-1 / 2}, n\right)=c_{1} h R d^{-1 / 2} x+\zeta_{K}(0),
$$

where $h$ is the class number of $K, R$ the regulator of $K$, and $c_{1}$ a constant depending only on $n$. If $K$ is an imaginary quadratic field, $\zeta_{K}(0)=c_{2} h$, where $c_{2}$ does not depend upon $d$; otherwise, $\zeta_{K}(0)=0$. From [5, p. 481] we have

$$
h R \leqq A d^{1 / 2} \log ^{n-1} d,
$$

where $A$ depends only on $n$. Thus,

$$
\left|Q^{*}\left(x, d^{-1 / 2}, n\right)\right| \leqq A x \log ^{n-1} d+\left|\zeta_{K}(0)\right|,
$$

where $\left|\zeta_{K}(0)\right| \leqq A d^{1 / 2} \log d$ if $K$ is an imaginary quadratic field. Also, from $[5$, p. 482],

$$
\sum_{\nu \leqq x} F(\nu) \leqq A x \log ^{n-1} d
$$

where $A$ depends only on $n$ and not on $x$ or $d$. In our theorem we can take $m=n$. Also, $\rho=-2 /(n+1)$. Choose $\eta=(n-1) /\{n(n+1)\}$. Thus,

$$
E\left(x, d^{-1 / 2}\right)=E\left(x, d^{-1 / 2}, n\right)=2 x^{(n-1) /(n+1)} d^{1 /(n+1)} \log ^{n-1} d .
$$

For $x^{2 /(n+1)} \leqq A d^{1 /(n+1)}$,

$$
x \log ^{n-1} d=x^{(n-1) /(n+1)} x^{2 /(n+1)} \log ^{n-1} d \leqq A E\left(x, d^{-1 / 2}, n\right) .
$$

For an imaginary quadratic field and $x^{2 / 3} \geqq A d^{1 / 3}$,

$$
\left|\zeta_{K}(0)\right| \leqq A d^{1 / 2} \log d=A d^{1 / 3} d^{1 / 6} \log d \leqq A E\left(x, d^{-1 / 2}, 2\right) .
$$

Thus, all the hypotheses of our theorem are satisfied, and we conclude that

$$
\sum_{\nu \leq x} F(\nu)-c_{1} h R d^{-1 / 2} x=O\left(x^{(n-1) /(n+1)} d^{1 /(n+1)} \log ^{n-1} d\right) .
$$

This problem has been considered by several authors. Ayoub [1], for an imaginary quadratic field, showed that the left side of (3) is 
$O\left(x^{1 / 3+\epsilon} d^{1 / 3+\epsilon}\right)+O\left(x^{\epsilon} d^{1 / 2+\epsilon}\right)$ for every $\epsilon>0$. Fögels has considered the problem and some generalizations. (See [2] and other papers of the author cited there.) The best results, however, were previously achieved by Landau [5] who showed that the left side of (3) is $O\left(x^{(n-1) /(n+1)} d^{1 /(n+1)} \log ^{n} d\right)$. Our result is better than Landau's by a factor of $\log d$. However, an examination of Landau's proof shows that his proof really yields the slightly better result that we give.

Our theorem also yields results for $L$-series of algebraic number fields.

The author is grateful to Paul Bateman and Raghavan Narasimhan for helpful conversations.

\section{REFERENCES}

1. Raymond G. Ayoub, On the coefficients of the zeta function of an imaginary quadratic field, Acta Arith. 13 (1967/68), 375-381. MR 37 \#1343.

2. E. Fogels, On the abstract theory of primes. III, Acta Arith. 11 (1965/66), 293-331. MR 33 \#5583.

3. K. Chandrasekharan and Raghavan Narasimhan, Functional equations with multiple gamma factors and the average order of arithmetical functions, Ann. of Math. (2) 76 (1962), 93-136. MR 25 \#3911.

4. E. Landau, Über die Anzahl der Gitterpunkte in gewissen Bereichen. (Zweite Abhandlung), Nachr. Ges. Wiss. Göttingen. Math. -Phys. Kl. 1915, 209-243.

5. - Verallgemeinerung eines Polyaschen Satzes auf algebraische Zahlkörpern, Nachr. Ges. Wiss. Göttingen. Math. -Phys. Kl. 1918, 478-488.

UNIVERSITY OF ILLINOIS, URBANA, ILLINOIS 61801 